\title{
Single Chains of Block Copolymers in Poor Solvents: Handshake, Spiral, and Lamellar Globules Formed by Geometric Frustration
}

\author{
Drew F. Parsons* and David R. M. Williams \\ Research School of Physical Sciences and Engineering, Australian National University, Canberra, ACT 0200, Australia
}

(Received 1 July 2007; published 30 November 2007)

\begin{abstract}
A single multiblock copolymer chain in poor solvent undergoes microphase separation within its own globule, driven by the same kind of forces operating in the bulk system. However, the necessity of packing a large $A B$ interface into a small volume leads to novel convoluted geometries. Long block lengths form a double droplet. Very short block lengths exhibit bulk behavior, forming a lamellar globule. With intermediate block lengths, the $A B$ interface buckles to form a hand shake or spiral dicluster. An order-disorder transition is reported for short block lengths.
\end{abstract}

DOI: 10.1103/PhysRevLett.99.228302

PACS numbers: 82.35.Jk, 36.20.Ey

The behavior of single polymer chains under different solvent conditions plays a central role in polymer physics and is crucial to understanding a wide variety of physical and biological problems. Our understanding of linear homopolymer chains where each monomer is identical is now reasonably thorough [1-4]. However, the same is not true for copolymers consisting of more than one kind of monomer. For bulk samples of copolymers, there are very precise and accurate theories, which match very well with detailed and exhaustive experiments [5]. For single copolymer chains in selective solvents, our knowledge is much poorer. This represents a definite lacuna, given the importance of copolymers in biology and indeed their fundamental importance in polymer physics. Random heteropolymers have been applied as models for protein folding [6,7], but the role of regular multiblock copolymers in this area has not yet been explored.

In this Letter, we make a preliminary examination of one of the simpler problems in single-chain copolymer behavior, a regular multiblock copolymer with two kinds of monomers where the solvent is poor for both. In the case of a homopolymer, the chain is understood to collapses to a structureless compact globule minimizing unfavorable contact between the solvent and the monomers. Introducing two kinds of monomers $A$ and $B$ immediately gives rise to a much richer behavior. In particular, microphase separation is possible, leading to various interesting internal structures. In some cases, they are analogous to those found in bulk samples of copolymers, but in many cases, the geometrical confinement implied by a globule gives rise to an array of new structures. The confined and buckled surfaces of these new geometries have a potential role in the production of highly-structured nanoscale objects.

In bulk block copolymer systems, microphase separation is driven by the enthalpic penalty of mixing; i.e., chemically different chain segments have energetic reasons for demixing. Complete phase separation is impossible because different chain segments are attached to one another; the result is microphase separation. In the strong segregation limit where the interfaces are sharp, the area per head group, $\sigma$ (in this case the $A B$ connections) is controlled by a balance between the interfacial tension, which favors small $\sigma$, or less interface, and the entropic chain deformation penalty which favors large $\sigma$. The same applies for single multiblock chains in poor solvent, with the added complication of confinement within a globule, which itself may change its external shape. These give rise to a novel regime where the chain tries to fit a certain amount of interface in a small volume, leading as we shall see, to buckled and tortuous interfaces.

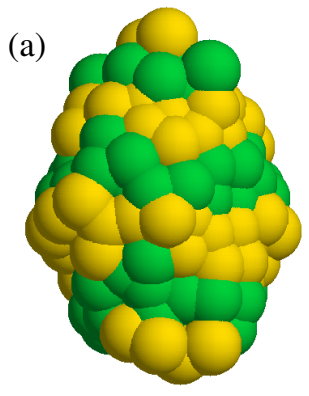

(b)

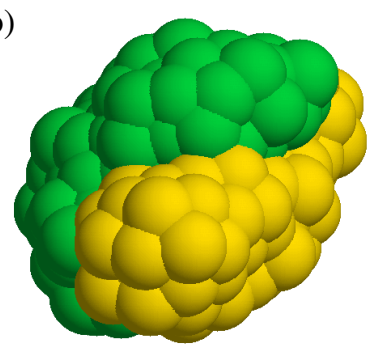

(c)

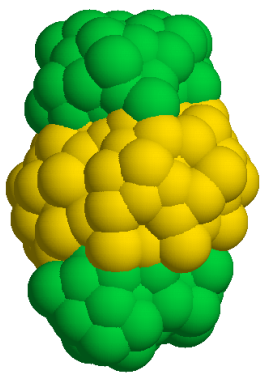

(d)

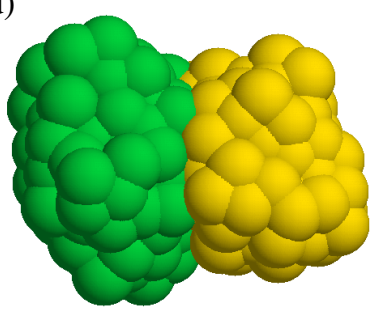

FIG. 1 (color online). Representative samples at different block sizes $m$, with total chain length $240, T=0.4$. Purely repulsive $A-B$ interaction are used. (a) Short blocks $m=2$. (b) Intermediate blocks $m=10$, "hand shake" dicluster. (c) Intermediate blocks $m=10$, triglobule. (d) Long blocks $m=30$. 
We examined multiblock copolymers using an offlattice ball-spring model $[4,8]$. Blocks were composed of $m$ monomers of type $A$ followed by $m$ monomers of type $B$, repeating the basic $A B$ block along the entire length of the chain. The total length $N$ of the polymer was 240 monomers, with the number of blocks changing as the number of monomers per block was varied. We report here the results for $m=2,10$, and 30, representing short, intermediate, and long block lengths, respectively.

Bond potentials were represented by a finitely extensible nonlinear elastic (FENE) potential and two-body interactions between monomers by a shifted truncated LennardJones potential [8]. We used Lennard-Jones energy well depths $\varepsilon_{A A}=\varepsilon_{B B}=1$ for interactions between monomers of the same type. We made the interaction between monomers of different type relatively repulsive by reducing their LJ well depth $\varepsilon_{A B}$ below 1, enabling phase separation between them. We also tested a purely repulsive $A B$ interaction potential, $U_{R}(l)=U_{\mathrm{LJ}}(l)+\varepsilon_{A B}$ for $l<2^{1 / 6} \sigma$ (zero at larger distances), using $\varepsilon_{A B}=1$ for this purely repulsive potential. Runs were performed using Wang-Landau Monte Carlo [8] to show the prevalence of different structures.

Representative structures formed at $T=0.6$ for $m=2$, 10, 30 are given in Fig. 1. A rough way of thinking about the system is as $N / 2 m A B$ diblocks, each with $2 m$ monomers. For a strongly-stretched system, balancing the surface tension and chain stretching free energy gives a preferred area per $A B$ connection of $\sigma_{0} \propto m^{1 / 3}$. As there are $N / m$ such joins in our system, the total preferred $A B$ area is $\Sigma_{0} \propto N m^{-2 / 3}$. Thus, as $m$ decreases, the number of blocks increases, and the preferred $A B$ area also increases. It is the $A B$ connectivity and its effect on the preferred area which leads to the interesting geometries seen. For large $m=30$, the required area is small, and the system forms a dumbbell-like dicluster, Fig. 1(d), similar to that which would be formed by a diblock copolymer. A collapsed diblock copolymer may be described as two separate immiscible liquid drops, characterized by their surface tensions with the solvent and with one another and attached to one another at only one point. As the number of blocks is increased, the double droplet model can no longer successfully describe the collapsed globule due to the increased number of connections between the $A$ and $B$ phases.

When the block size is decreased down to intermediate lengths, say to $m=10$, the preferred $A B$ interface area becomes larger. At this point, free energy minimization is geometrically frustrated by the competing requirement that the $A B$ interface be large but that it must fit inside a spherical globule to minimize contact with the solvent. To form the extra $A B$ interface, the system then does one of two things. It can form a tricluster, Fig. 1(c), in which case two completely separate $A B$ interfaces are made. Alternatively, a single almost spherical globule is formed in order to minimize contact with the surrounding solvent,

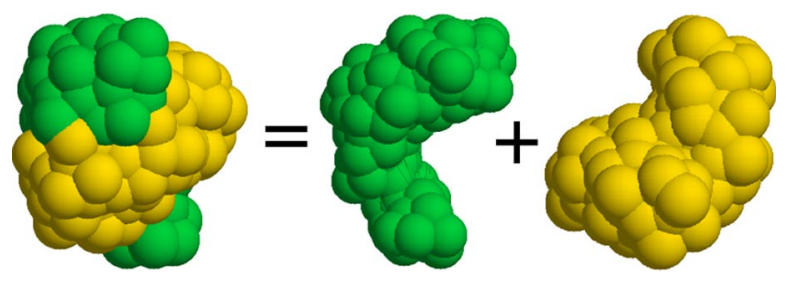

FIG. 2 (color online). Handshake dicluster at intermediate block length $(m=10)$ for $\varepsilon_{A B}=0.1$, split into component phases. Total chain length 240 monomers.

with the two $A$ and $B$ phases forced to coexist in a "tennis ball" or "handshake" conformation, shown in Fig. 1(b). This handshake dicluster is interesting since the two phases do not simply form spherical hemispheres with a plane interface between them. Because of the necessity of forming a large $A B$ interface mentioned above, the two phases are only able to form a single sphere together by wrapping around one another the way two hands do in a handshake. The $A-B$ interface is revealed by splitting the two phases apart in Fig. 2. At $\varepsilon_{A B}=0.1$, the ratio of triglobules to handshake diclusters is about $6: 1$. When phase separation is maximized by using the purely repulsive $A-B$ interaction potential, then a smaller amount of the handshake dicluster [Fig. 1(b)] is formed, with the triglobule:dicluster ratio rising to $10: 1$.

At short block lengths $(m=2)$ Fig. 1(a), the geometric frustration becomes even more significant. $A$ much larger $A B$ interface is required, and the system forms a handful of flat layers. The separate layers may be observed in the split view, Fig. 3. This phase separation into flat layers is reminiscent of the formation of the lamellar phase by a block copolymer in bulk. That is, the bulk lamellar phase is obtained in the limit where the block size becomes small relative to the overall size of a system.

Reducing the relative degree of repulsion by using $\varepsilon_{A B}=0.5$, pure phase separation does not occur in the short $m=2$ system, and a disordered globule is formed instead (at $T=0.81$ ), Fig. 4. This disordered globule is a fifth qualitatively different structure formed by multiblock copolymers in poor solvent.

Diminishing phase separation by taking $\varepsilon_{A B}=0.5$, the intermediate system $(m=10)$ is able to more readily form the handshake dicluster, with the ratio of triglobules to diclusters, being $6: 1$ at $\varepsilon_{A B}=0.1$, changing to $1: 1$ at

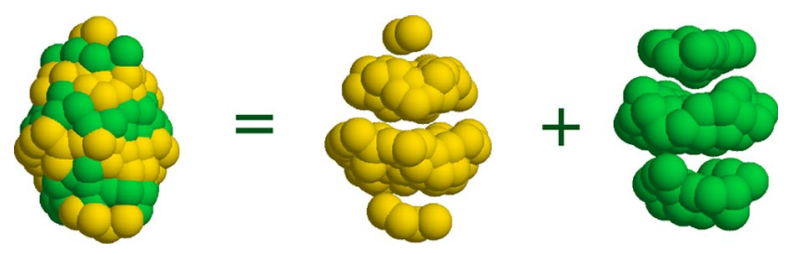

FIG. 3 (color online). Layered globule at short block length $(m=2)$ for purely repulsive $A B$ interactions. Globule split into component phases. Total chain length 240 monomers. 


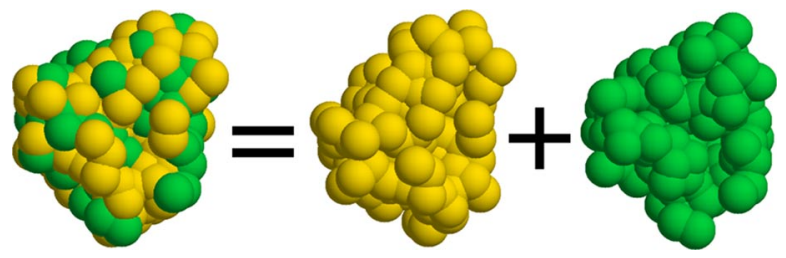

FIG. 4 (color online). Disordered globule for short block length $(m=2)$ at $\varepsilon_{A B}=0.1$ collected for $T=0.81$, above the strong heat capacity peak at $T=0.54$, split into component phases. Total chain length 240 monomers.

$\varepsilon_{A B}=0.5$. With a greater surface area permitted between the $A$ and $B$ blocks, the handshake configuration is able to bend along several more turns forming a spiral structure, see Fig. 5. We suggest the handshake and spiral diclusters form an analogue of the gyroid phase observed in block copolymer melts [9].

The Wang-Landau technique yields information over a range of temperatures. It allows us to examine transitions as the chain is cooled, which manifest as peaks in the heat capacity curve, $C_{V}=d\langle E\rangle / d T$. The heat capacity curve for short block lengths $(m=2)$ at $\varepsilon_{A B}=0.1$ is shown in Fig. 6. A strong transition in the globule may be seen at region A near $T=0.54$, just above the point $T=0.4$ used for the sample in Fig. 1(a) but below the coil-globule transition seen near $T=1.1$. The trough at region $B(T=$ 0.81 ) on the other side of the peak is represented by the disordered globule shown in Fig. 4, contrary to the layered globule seen at $T=0.4$ [Figs. 1(a) and 3]. We therefore identify this peak as an order-disorder transition occurring inside the collapsed globule for copolymers with short block length. The smaller peaks in this heat capacity curve are related to more subtle rearrangements inside the globule such as compactification [4]. The coil-globule transition of the multiblock copolymer at $T=1.1$ is somewhat depressed from the value of $T=1.7$ seen for an equivalent homopolymer of the same length [4].

The heat capacity curve for intermediate block lengths ( $m=10)$ at $\varepsilon_{A B}=0.1$ is shown in Fig. 7. We identify four major regions in this curve, labeled $A, B, C$, and $D$. Region A corresponds to the low temperature state represented in Figs. 1(b) and 1(c), while region $D$ is the expanded coil. The identity of regions $B$ and $C$ is illuminated by the cluster distribution in Fig. 8, describing the relative pro-

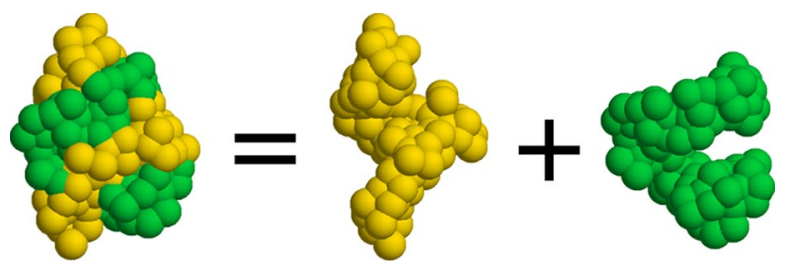

FIG. 5 (color online). Spiralling dicluster at intermediate block length $(m=10)$ for $\varepsilon_{A B}=0.5$, split into component phases. Total chain length 240 monomers.

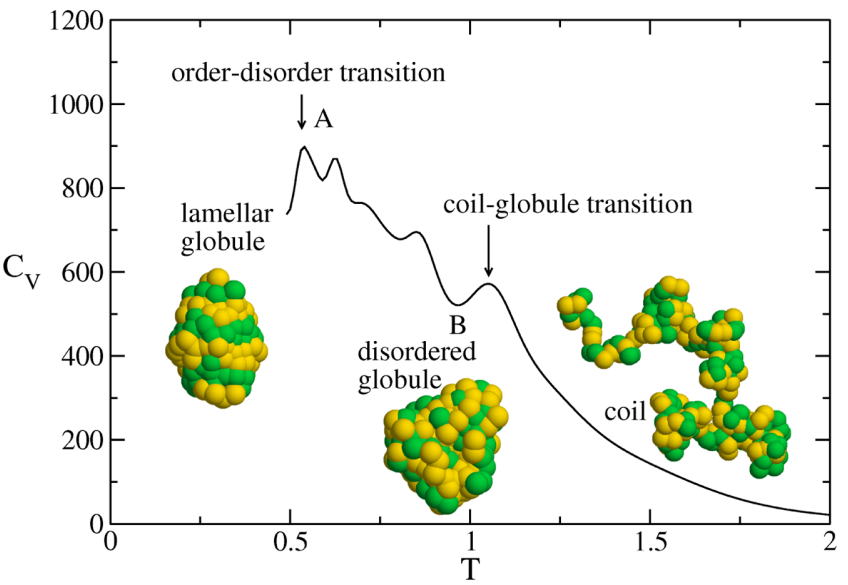

FIG. 6 (color online). Heat capacity for short block length $(m=2)$ at $\varepsilon_{A B}=0.1$. The peak at $A$ marks the order-disorder transition, $B$ marks the stable region of the disordered globule.

portion of different numbers of clusters as a function of temperature, where each separated blob of monomer $A$ or $B$ is counted as one cluster, using a cluster counting algorithm [10]. A cluster is a set of monomers of the same type located no more than $2 \sigma$ from its nearest neighbor, $\sigma$ being the Lennard-Jones length parameter. Each region $B$ and $C$ matches a peak in the cluster distribution, corresponding to 4-clusters and 5-clusters, respectively. We therefore interpret the peaks in the heat capacity intermediate block lengths as transition peaks corresponding to the progressive collapse of the copolymer, from expanded coil to 5cluster (globule), to 4-cluster and finally to the tricluster and dicluster seen in Fig. 1.

We saw above that when the block length is sufficiently reduced (to around $m=10$ in our case), a buckled hand shake dicluster forms, distorting the $A B$ interface. We expect this would occur when the available unbuckled $A B$ area $\sigma$ per $A B$ join (the flat surface of a hemisphere) becomes some fraction of the natural $A B$ area $\sigma_{0}$, which

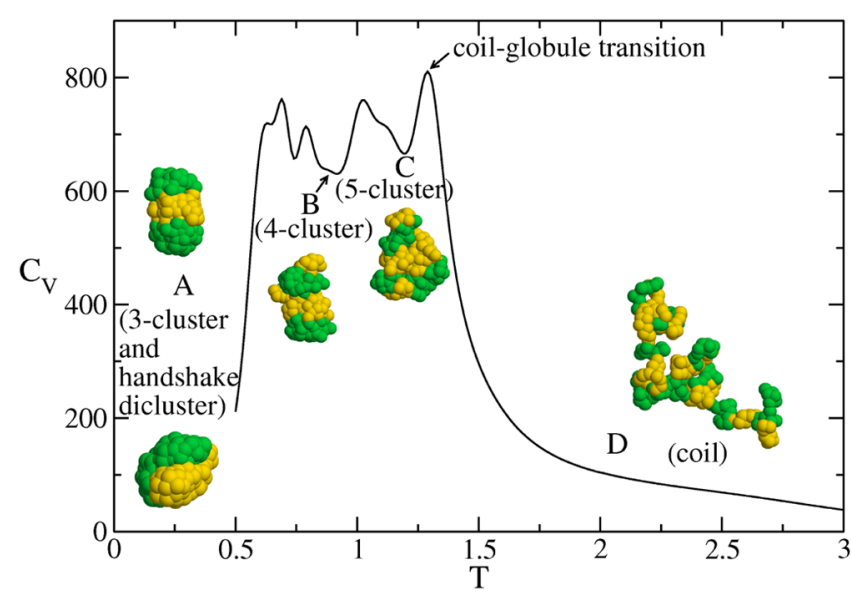

FIG. 7 (color online). Heat capacity for intermediate block length $(m=10)$ at $\varepsilon_{A B}=0.1$. 


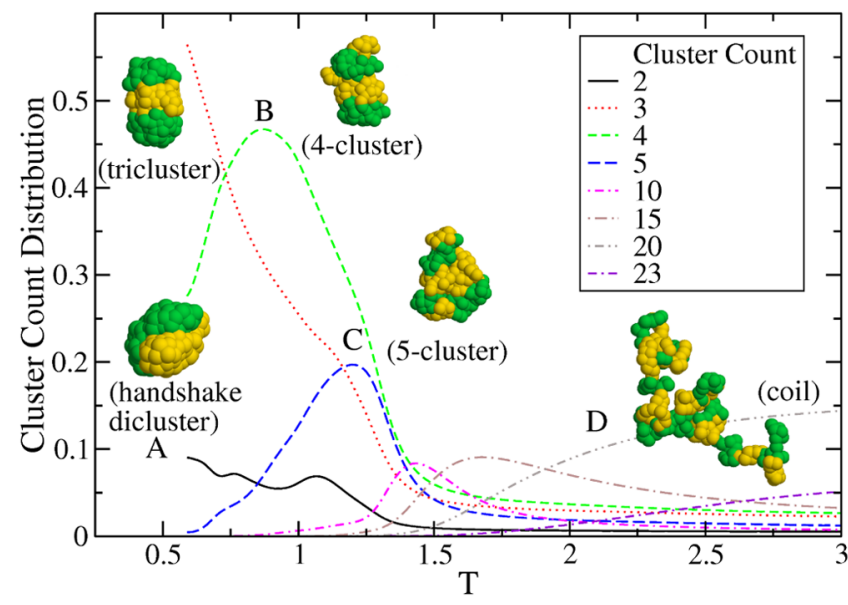

FIG. 8 (color online). Cluster count distribution for intermediate block length $(m=10)$ at $\varepsilon_{A B}=0.1$. Describes the relative proportion of various cluster counts at each temperature. Regions $A, B, C$, and $D$ correspond to stable regions in the heat capacity, Fig. 7. For clarity, only selected cluster counts are shown.

for instance would be present in a bulk sample, i.e., when $\sigma=\epsilon \sigma_{0}$ where $\epsilon<1$. We can treat the unbuckled system as if it were an ordinary monolayer of diblocks, each with $m$ monomers. The free energy of one such diblock is given by the sum of the surface energy of the $A B$ interface and the stretching of the chain to some height $H, F=$ $g k T \sigma / a^{2}+\alpha k T H^{2} /\left(m a^{2}\right)$, where $g$ and $\alpha$ are parameters of order unity. For monomers of volume $\frac{4}{3} \pi a^{3}, H$ and $\sigma$ are related by $H \sigma=\frac{4}{3} \pi a^{3} m$. Minimizing over $\sigma$ gives the "natural" area $\sigma_{0}=\left[32 \pi^{2} \alpha /(9 g)\right]^{1 / 3} a^{2} m^{1 / 3}$. On the other hand, the area per $A B$ connection in the unbuckled state is given by $\sigma=\pi R^{2} m / N$, where $R^{3}=N a^{3}$, by conservation of monomer volume. Writing $\sigma=\epsilon \sigma_{0}$ then gives a critical block length for the handshake cluster of $m=\epsilon^{3 / 2}[32 \alpha /(9 \pi g)]^{1 / 2} N^{1 / 2}$. We can go a little further and get a handle on the parameter $\epsilon$ by noticing that the buckling to the handshake cluster is closely related to the Helfrich-Hurault transition [11] which occurs in smectic systems under extension. The critical extension $H$ for diblock systems is related to the natural length $H_{0}$ by [12] $\left(H-H_{0}\right) / H_{0}=\pi H_{0} /(2 H)$. Converting from height to area gives us $\epsilon=(-1+\sqrt{1+2} \pi) / \pi$ so that the critical block length is then $m \approx 0.42 N^{1 / 2} \sqrt{\alpha / g}$. In our case, $N=$ 240 and setting $g=\alpha=1$ gives $m \approx 7$, very close to the value $m=10$ where we see a clear handshake cluster. We can also say that the transition occurs when the preferred bulk diblock height $H_{0}$ is related to the globule radius by $H_{0} \approx 0.7 R$. These calculations make rather sweeping approximations about the geometry, but it is likely they are close to the truth in all but the numerical prefactors.

In summary, we have presented results for the geometries one finds for single-chain multiblock copolymers in poor solvents. The driving forces in this case are similar to those in the more traditional bulk problem. The geometries we find are sometimes similar (lamellae for instance), and sometimes very different as in the case of the handshake cluster. The major interest is how confinement within a globule, which in our case is a flexible kind of confinement, affects chain morphology. The overall globular confinement affects the $A B$ interface shape, but the fact that there is a tortured $A B$ interface in the globule in turn affects the overall globular shape. It would be rash to claim that our study is anything like complete. We have for instance no phase diagram and we have considered only a singlechain length. Many questions have in fact only been touched on, such as how the order-disorder transition occurs in this system. These are obviously themes for future investigation.

*Drew.Parsons@anu.edu.au

[1] C. Wu and X. Wang, Phys. Rev. Lett. 80, 4092 (1998).

[2] C. Williams, F. Brochard, and H. L. Frisch, Annu. Rev. Phys. Chem. 32, 433 (1981).

[3] F. Rampf, W. Paul, and K. Binder, Europhys. Lett. 70, 628 (2005).

[4] D. F. Parsons and D. R. M. Williams, J. Chem. Phys. 124, 221103 (2006).

[5] F. Bates and G. Fredrickson, Annu. Rev. Phys. Chem. 41, 525 (1990).

[6] A. Khokhlov, A. Semenov, and A. Subbotin, Eur. Phys. J. E 17, 283 (2005).

[7] V.S. Pande, A. Y. Grosberg, and T. Tanaka, Rev. Mod. Phys. 72, 259 (2000).

[8] D. F. Parsons and D. R. M. Williams, Phys. Rev. E 74, 041804 (2006).

[9] G. Grason, Phys. Rep. 433, 1 (2006).

[10] E. M. Sevick, P. A. Monson, and J. M. Ottino, J. Chem. Phys. 88, 1198 (1988).

[11] P. G. de Gennes and J. Prost, The Physics of Liquid Crystals (Clarendon Press, Oxford, 1993), 2nd ed..

[12] Z.-G. Wang, J. Chem. Phys. 100, 2298 (1994). 